\title{
Simplification of hepatitis $C$ testing: a time to act
}

\author{
Mario Poljak ${ }^{1 凶}$ \\ ${ }^{1}$ Institute of Microbiology and Immunology, Faculty of Medicine, University of Ljubljana, Ljubljana, Slovenia.
}

\begin{abstract}
Hepatitis C virus (HCV) affects 71 million people worldwide. The development of reliable diagnostic tools in the last 2 decades and recent implementation of highly potent and safe antiviral drug combinations have paved the way to potential elimination of HCV as a public health threat by 2030 . This article briefly discusses current and upcoming solutions for simplification of HCV testing taking into account the most recent guidance documents issued by major professional societies. The general consensus is that all patients with suspected HCV infection should be tested for anti-HCV antibodies as a first-line diagnostic test. Some anti-HCV rapid diagnostic tests have already attained the performance of standard anti-HCV enzyme immunoassays. If anti-HCV antibodies are detected, the presence of HCV RNA or HCV core antigen should be determined to identify patients with ongoing infection. Several innovative devices for detecting HCV in serum or plasma are in the late stages of development and are based on loop-mediated isothermal amplification, smartphone-operated instruments, biosensors, lab-on-a-chip solutions, paper-based microfluidics, and CRISPR-Cas. An important solution for low- and middle-income countries is the availability of HCV tests that could perform equally reliably from whole blood and dried blood spots as well as from serum or plasma. Another interesting diagnostic concept for these countries is near-to-patient diagnostics using mobile microbiological laboratories, following either the lab-on-a-drone or rent-apoint-of-care-test concepts. Using current and upcoming diagnostic approaches, the elimination of HCV is plausible, but in several countries this is probably not possible within the timeframe suggested by the World Health Organization. Two different elimination approaches have already been successfully tested in real-life conditions: micro-elimination and macro-elimination. The microelimination approach has resulted in successful elimination in specific population subgroups in some high-income countries. In at least two countries, Georgia and Egypt, a macro-elimination approach has shown impressive real-life results relatively quickly.
\end{abstract}

Keywords: hepatitis C, HCV, diagnosis, testing, simplification

Received: 1 June 2020 | Returned for modification: 15 July 2020 | Accepted: 6 August 2020

\section{Introduction}

Chronic infection with hepatitis C virus (HCV) affects 71 million people worldwide and accounts for 9.7 million disability-adjusted life years annually $(1,2)$. Global mortality related to its complications (cirrhosis, liver failure, and hepatocellular carcinoma) is estimated at 580,000 person per year $(1,2)$. Although initially only rarely connected with sexual transmission, recent evidence of HCV outbreaks in Europe, the United States, and Australia among men who have sex with men (MSM) who denied injecting drugs clearly showed that HCV should be considered an emerging sexually transmissible agent (3). In addition, the high reinfection rates among MSM who cleared HCV infection spontaneously or who were successfully treated further underline the importance of sexual behavior in HCV transmission (3).

The development of reliable virological diagnostic and monitoring tools in the last 2 decades and the recent implementation of highly potent, safe, well-tolerated direct-acting antiviral (DAA) drug combinations with a high barrier to resistance have paved the way to potential elimination of HCV as a public health threat by 2030 (4). Although HCV elimination can now be envisaged in several countries, HCV remains a global public health concern. Many parts of the world are lacking political will and advocacy, economic support, or adequate implementation infrastructure and are in urgent need of simplified screening, diagnosis, linkage to care, treatment, and monitoring procedures to achieve this ambitious elimination goal $(5,6)$. To eliminate HCV globally, we desperately need creative solutions throughout the complete cascade of care.
This narrative review briefly discusses current and upcoming solutions for simplification of HCV testing, taking into account the most recent guidance documents issued by the European Association for the Study of the Liver (EASL) (7), the American Association for the Study of Liver Diseases (AASLD) jointly with the Infectious Diseases Society of America (IDSA) (8), and the World Health Organization (WHO) (4).

\section{How to screen for HCV infection}

There is a general consensus that all patients with suspected HCV infection should be tested for anti-HCV antibodies in serum or plasma as a first-line diagnostic test $(7,8)$. Highly standardized, reliable, and rapid anti-HCV enzyme immunoassays performed on automated analyzers with random access modality are the standard of care in many countries. However, in many parts of the world more simplified screening approaches are needed, such as affordable rapid diagnostic tests (RDTs) using serum, plasma, fingerstick whole blood, or crevicular fluid (saliva) as matrices that can be used instead of standard enzyme immunoassays at the patient's care site to facilitate screening and improve access to care (7). An ideal RDT must meet the WHO “ASSURED" criteria (9), and it must be easy to use with minimal training. Some anti-HCV RDT tests have already attained the performance level of standard enzyme immunoassays (10); for more details, see recent reviews and a meta-analysis on the topic $(11,12)$. Good examples of important recent technical achievements in the field are the development of RDT for simultaneous detection of hepatitis A, B, and C viruses (13) 
and the development of a simple paper-based device allowing separation of plasma from whole blood without the use of centrifugation (14).

In addition to anti-HCV, screening for HCV RNA in serum or plasma is now inevitable in transfusion settings, and HCV RNA testing should be part of the initial evaluation in cases of suspected acute hepatitis $\mathrm{C}$, in immunocompromised patients, and in patients on hemodialysis (7). In some screening settings where a laboratory mainly serves high-risk populations such as intravenous drug users, 24-mini-pool HCV RNA screening of anti-HCV negative samples has been shown to be valuable and cost-effective $(15,16)$.

\section{How to proceed after anti-HCV positivity}

If anti-HCV antibodies are detected, the presence of HCV RNA in serum or plasma should be determined to identify patients with ongoing infection, preferably using a sensitive molecular method with a lower limit of detection $(\leq 15 \mathrm{IU} / \mathrm{ml})$ and following the reflex testing approach to increase linkage to care (7). Highly standardized and reliable HCV RNA assays performed on fully automated sample-to-result fashion analyzers with sample number flexibility and the ability to perform multiple tests concordantly are now becoming the standard of care in many countries, replacing older batch-based testing platforms. Alternatively, smaller and portable molecular devices such as GeneXpert (Cepheid, Sunnyvale, CA) (17-19) or Genedrive (Genedrive Diagnostics, Manchester, UK) can be used for reliable, highly sensitive, and rapid detection of HCV RNA (11, 20). In addition, there are several commercial portable molecular devices in the launching stage or late stage of development, and some of them are even suitable for use in extreme environmental conditions $(20,21)$. The goal with portable molecular instruments is to have a self-contained, fully integrated, sampleto-report device that accepts raw, untreated specimens (preferably whole blood), performs all of the molecular steps, and provides interpreted test results in less than an hour.

However, all these solutions are not easily affordable for lowand middle-income countries (LMIC), and so new molecular assays that facilitate easy and inexpensive access and linkage to care are desperately needed. In 2018, EASL recommended the use of qualitative, not necessarily quantitative, HCV RNA assays with a limit of detection $\leq 1,000 \mathrm{IU} / \mathrm{ml}(3.0 \mathrm{log} \mathrm{IU} / \mathrm{ml})(7)$. In the settings where these assays will be used, the exceptionally low risk of a false-negative result in a small percentage of infected individuals (22) is outweighed by the benefit of scaling up access to diagnosis and care $(11,23)$. Namely, a recent study performed on a global dataset showed that a test with a limit of detection of $1,318 \mathrm{IU} / \mathrm{ml}$ would identify $97 \%$ of viremic HCV infections among almost all populations (24). Such future assays with lower but acceptable sensitivity and based on already existing molecular device prototypes will use instruments based on loop-mediated isothermal amplification (LAMP) technology $(25,26)$, smartphone-operated instruments (26), biosensors (27), lab-on-a chip solutions (28), paper-based microfluidics solutions $(29,30)$, CRISPR-Cas $(31,32)$, and even wearable devices (33). All these technical solutions should have a low frequency of invalid results and an affordable price. Among all technical solutions, those based on smartphones are most appealing because about $80 \%$ of the world population uses smartphones as of 2020, offering an ideal interface for different portable medical instruments.

Although available for almost two decades (34), and despite strong supporting scientific evidence (22-24, 35), as well as guide- line recommendations (7), detection of HCV core antigen in serum or plasma in settings where HCV RNA assays are not available and/ or not affordable to identify patients with ongoing infection is still slowly making its way into wider implementation. One of the main reasons is marginally lower analytical sensitivity of HCV core antigen detection in comparison to conventional HCV RNA assays. However, as already mentioned in real-life cohorts, the prevalence of low HCV viremia in untreated individuals is below $5 \%$, and so the benefits of the HCV core antigen assay as a rapid, low-cost, and potential point-of-care test in population-based screening still outweigh the risk of a small number of false negatives in a subset of patients (23). A recent study showed that a great majority of patients with low viremia are patients with cirrhosis and excessive alcohol use, allowing stratified testing with HCV core antigen or HCV RNA to reliably identify patients with ongoing infection (22).

\section{Alternative specimen types and alternative transport of samples and point-of-care devices}

An important solution for LMICs is the availability of HCV diagnostic tests that can perform equally reliably from whole blood and dried blood spots (DBSs) as well as from serum or plasma (7). The main advantage of DBSs is to allow storage of desiccated blood that can be easily transported to reference centers, where state-of-the-art serological and molecular tests are available (11). Although standardization and better automation of DBS handling are needed, recent systematic reviews and meta-analyses showed only marginally inferior performance of anti-HCV testing, HCV RNA testing, and HCV genotype determination from DBSs in comparison to plasma or serum, with the exception of HCV core antigen testing $(11,36-39)$.

Alternatively, whole blood samples can be rapidly and easily transported to reference centers in LMICs using drones. In the last decade, the use of drones has gradually spread into various healthcare settings (40). Rwanda and Tanzania recently launched a large delivery network with more than 1,00o health facilities connected by drones parachuting blood, vaccines, and malaria and HIV/AIDS drugs (40). Specimen transport by drones between several hospitals and a central laboratory has been routinely used in Lugano (Switzerland) and in Antwerp (Belgium) since 2018 and 2019, respectively (40).

Another interesting concept for LMICs is near-to-patient or point-of-care (POC) diagnostics using mobile microbiological laboratories. This can be achieved following the lab-on-a-drone (41) or rent-a-POC-test concepts (40). In the lab-on-a-drone prototype, an innovative and robust sample preparation device coupled with an isothermal amplification instrument and smartphone for measuring readouts and recording results are all attached to a quadcopter drone (41). In addition, the drone's propellers are removable and replaceable with locally $3 \mathrm{D}$-printed centrifuge rotors of different shapes, capable of speeds up to 10,000 rpm and performing column-based extraction of high-quality RNA/DNA (40). The recently proposed rent-a-POC-test concept (40) follows the principle of a drone network pilot program built to deliver automated external defibrillators (42). The rent-a-POC-test concept is based on a central microbiology laboratory responsible for validation, calibration, and maintenance of POC instruments, their delivery, and quality control. Drones can be deployed to deliver on-demand portable POC instruments and test reagents to remote healthcare facilities, eliminating the need for local storage of instruments or tests and associated maintenance costs (40). In an 
HCV elimination scenario, one could imagine drones loaded not only with portable POC instruments able to detect $\mathrm{HCV}$ viremic patients but also with prepacked DAAs for 8 to 12 weeks' treatment allowing an HCV test-and-treat strategy to be implemented in the most remote parts of the world.

\section{Is large-scale screening for HCV infection achievable?}

Using the diagnostic approaches described above, I strongly believe that large-scale elimination of HCV is plausible, but in several countries this is probably not possible within the timeframe suggested by the WHO. Two different elimination approaches have already been successfully tested in real-life conditions: "micro-elimination" and "macro-elimination" (6).

The majority of high-income countries, including Slovenia, recently launched a micro-elimination strategy as a first step toward universal national elimination. This practical and comprehensive concept, which breaks down national goals into smaller goals, uses a population segmentation approach in order to tailor concentrated elimination efforts to specific population subgroups; for example, individuals with HIV, patients with hemophilia, $\beta$-thalassemia major, decompensated cirrhosis, or organ transplantation, patients treated with hemodialysis, (ever) injection drug users, MSM, migrants from high endemic countries, healthcare workers, and prisoners (43). Micro-elimination, by way of targeting smaller and clearly delineated HCV risk groups, allows faster and more efficient delivery of interventions. For this reason, micro-elimination as a "bottom-up" approach may be a more feasible and efficient path to nationwide HCV elimination in some settings (43).

In contrast to micro-elimination strategies, the macro-elimination approach does not focus on a specific group(s) but is instead directed toward the entire population of a country or a large segment of it (6). Macro-elimination is initiated with either a massscreening program of the entire population or a cohort screening of a segment of the population with a historically high prevalence of HCV infection (6). In at least two countries, Georgia and Egypt, a macro-elimination approach has shown impressive real-life results relatively quickly (44-47).

Georgia, with a high prevalence of HCV infection, launched the world's first national hepatitis C elimination program in April 2015 (46). A key strategy was identifying, treating, and curing the estimated 150,000 HCV-infected people living in the country. As of the end of 2018 , more than one million adults ( $40 \%$ of the adult population) had been screened for anti-HCV antibodies, of whom $8.9 \%$ tested positive (47). Of the individuals that tested anti-HCV positive, $80 \%$ were tested for the presence of HCV RNA, of whom $85.3 \%$ tested positive for active HCV infection. A total of 52,576 people with active HCV infection initiated treatment, and 48,879 had completed their course of treatment as of the end of 2018 (47). Sustained virologic response was achieved in $98.5 \%$ of individuals treated. Reflex testing for HCV core antigen, implemented in March 2018, increased the rates of monthly viremia testing by 97.5\% among those that screened positive for anti-HCV (47). Between 2015 and 2018, over one-third of people presumably living with HCV in Georgia were detected and linked to care and treatment; however, identification and linkage to care of the remaining individuals with HCV infection is challenging (47).

Egypt has the highest rate of hepatitis C infection in the world. Ten years ago, it was estimated that nearly $15 \%$ of Egyptians were infected; the majority of them were infected during the country's decades-long fight against schistosomiasis (48). On October 1st, 2018, Egypt launched the "10o Million Healthy Lives" program. The goal was to screen all Egyptians 12 years of age or older for HCV infection, hypertension, diabetes, and obesity, and to offer free treatment or counseling for people that tested positive (44). Of a target population of 62.5 million, a total of 50 million $(80 \%)$ spontaneously participated in screening between October 2018 and April 2019. More than 4.6\% of them had anti-HCV antibodies, of whom $76.5 \%$ had active viremia (45). Sustained virologic response was achieved in $98.8 \%$ of individuals treated. Due to a very strong and transparent negotiation policy, the total cost of the HCV testing and treatment amounted to only \$207.1 million. Negotiations led to a price reduction to only $\$ 0.58$ per screening test, $\$ 4.80$ per HCV RNA test, and $\$ 85$ for 12 weeks of sofosbuvir plus daclatasvir treatment (45). The cost of identifying a patient with HCV viremia was $\$ 85.41$, and the cost of identifying and curing a patient was $\$ 130.62$ (45).

\section{Acknowledgments}

I am grateful to Anja Šterbenc for her help with the references and technical support. This work was presented in part at the European Society of Clinical Microbiology and Infectious Diseases (ESCMID) graduate education course "Elimination of viral hepatitis: are we ready?” which was held in Ljubljana, Slovenia (September 27th-28th, 2019) in the form of the oral presentation "How do we diagnose the undiagnosed? A challenge for simplification.”

\section{References}

1. Blach S, Zeuzem S, Manns M, Altraif I, Duberg AS, Muljono DH, et al. Global prevalence and genotype distribution of hepatitis $C$ virus infection in 2015: a modelling study. Lancet Gastroenterol Hepatol. 2017;2:161-76.

2. World Health Organization (WHO). Global Hepatitis Report 2017 [Internet]. [Accessed on May 1, 2020]. Available from: https://www.who.int/hepatitis/publications/global-hepatitis-report2017/en/.

3. Nijmeijer BM, Koopsen J, Schinkel J, Prins M, Geijtenbeek TB. Sexually transmitted hepatitis $C$ virus infections: current trends, and recent advances in understanding the spread in men who have sex with men. J Int AIDS Soc. 2019;22: e25348.

4. World Health Organization (WHO). Global health sector strategy on viral hepatitis 2016-2021. [Accessed on May 1, 2020]. Available from: https://apps.who.int/ iris/bitstream/handle/10665/246177/WHO-HIV-2016.06-eng.pdf?sequence=1.

5. Pawlotsky JM. Interferon-free hepatitis C virus therapy. Cold Spring Harb Perspect Med. 2020: a036855
6. Matičič M, Lombardi A, Mondelli MU, Colombo M; ESCMID Study Group for Viral Hepatitis (ESGVH). Elimination of hepatitis C in Europe: can WHO targets be achieved? Clin Microbiol Infect. 2020;26:818-23.

7. European Association for the Study of the Liver. EASL recommendations on treatment of hepatitis C 2018. J Hepatol. 2018;69:461-511.

8. Ghany MG, Marks KM, Morgan TR, Wyles DL, Aronsohn Al, Bhattacharya D, et al. Hepatitis C guidance 2019 update: AASLD-IDSA recommendations for testing, managing, and treating hepatitis $C$ virus infection. Hepatology. 2020;71:686721.

9. Peeling RW. Testing for sexually transmitted infections: a brave new world? Sex Transm Infect. 2006;82:425-30.

10. Poiteau L, Soulier A, Lemoine M, Mohammed Z, Wlassow M, Rwegasha J, et al. Performance of a new rapid diagnostic test for the detection of antibodies to hepatitis C virus. J Virol Methods 2018;261:153-5. 
11. Chevaliez S, Pawlotsky JM. New virological tools for screening, diagnosis and monitoring of hepatitis $B$ and $C$ in resource-limited settings. J Hepatol. 2018;69:916-26.

12. Khuroo MS, Khuroo NS, Khuroo MS. Diagnostic accuracy of point-of-care tests or hepatitis C virus infection: a systematic review and meta-analysis. PLoS One. 2015;10:e0121450.

13. Kweon OJ, Lim YK, Kim HR, Kim TH, Lee MK. Analytical performance of newly developed rapid point-of-care test for the simultaneous detection of hepatitis A, $B$, and C viruses in serum samples. J Med Virol. 2019;91:1056-62.

14. Carmona S, Seiverth B, Magubane D, Hans L, Hoppler M. Separation of plasma from whole blood by use of the cobas plasma separation card: a compelling alternative to dried blood spots for quantification of HIV-1 viral load. J Clin Microbiol. 2019;57:e01336-18.

15. Seme K, Mocilnik T, Fujs K, Babic DZ, Todorović A, Fras-Stefan T, et al. Twentyfour mini-pool HCV RNA screening outside a blood transfusion setting: results of a 2-year prospective study. J Virol Methods. 2007;140:218-21.

16. Seme K, Mocilnik T, Poljak M. Twenty-four mini-pool HCV RNA screening in routine clinical virology laboratory setting: a six-year prospective study. J Virol Methods 2011;171:303-5.

17. Wlassow M, Poiteau L, Roudot-Thoraval F, Rosa I, Soulier A, Hézode C, et al. The new Xpert HCV viral load real-time PCR assay accurately quantifies hepatitis C virus RNA in serum and whole-blood specimens. J Clin Virol. 2019;117:80-4.

18. Gupta E, Agarwala P, Kumar G, Maiwall R, Sarin S. Point-of-care testing (POCT) in molecular diagnostics: performance evaluation of GeneXpert HCV RNA test in diagnosing and monitoring of HCV infection. J Clin Virol. 2017;88:46-51.

19. McHugh MP, Wu AHB, Chevaliez S, Pawlotsky JM, Hallin M, Templeton KE. Multicenter evaluation of the Cepheid Xpert Hepatitis C Virus Viral Load assay. J Clin Microbiol. 2017;55:1550-6.

20. Židovec Lepej S, Poljak M. Portable molecular diagnostic instruments in microbiology: current status. Clin Microbiol Infect. 2020;26:411-20.

21. Drain PK, Dorward J, Bender A, Lillis L, Marinucci F, Sacks J, et al. Point-of-care HIV viral load testing: an essential tool for a sustainable global HIV/AIDS response. Clin Microbiol Rev. 2019;32:e0oo97-18.

22. Bertisch B, Brezzi M, Negro F, Müllhaupt B, Ottiger C, Künzler-Heule $P$, et al. Very low hepatitis $C$ viral loads in treatment-naive persons: do they compromise hepatitis $C$ virus antigen testing? Clin Infect Dis. 2020;70:653-9.

23. Mathur $P$, Kottilil S. Hepatitis $C$ core antigen testing: still an effective diagnostic method for global elimination of hepatitis C. Clin Infect Dis. 2020;70:674-5

24. Freiman JM, Wang J, Easterbrook PJ, Horsburgh CR, Marinucci F, White LF, et al. Deriving the optimal limit of detection for an HCV point-of-care test for viraemic infection: analysis of a global dataset. J Hepatol. 2019;71:62-70.

25. Curtis KA, Rudolph DL, Morrison D, Guelig D, Diesburg S, McAdams D, et al. Single-use, electricity-free amplification device for detection of HIV-1. J Virol Methods. 2016;237:132-7.

26. Ong DSY, Poljak M. Smartphones as mobile microbiological laboratories. Clin Microbiol Infect. 2020;26:421-4.

27. Ahmed A, Rushworth JV, Hirst NA, Millner PA. Biosensors for whole-cell bacterial detection. Clin Microbiol Rev. 2014;27:631-46.

28. Gurrala R, Lang Z, Shepherd L, Davidson D, Harrison E, McClure M, et al. Novel $\mathrm{pH}$ sensing semiconductor for point-of-care detection of HIV-1 viremia. Sci Rep. 2016;6:36000.

29. Reboud J, Xu G, Garrett A, Adriko M, Yang Z, Tukahebwa EM, et al. Paper-based microfluidics for DNA diagnostics of malaria in low resource underserved rural communities. Proc Natl Acad Sci USA. 2019;116:4834-42.
30. Tsaloglou MN, Nemiroski A, Camci-Unal G, Christodouleas DC, Murray LP, Con nelly JT, et al. Handheld isothermal amplification and electrochemical detection of DNA in resource-limited settings. Anal Biochem. 2018;543:116-21.

31. Gootenberg JS, Abudayyeh 00, Lee JW, Essletzbichler P, Dy AJ, Joung J, et al. Nucleic acid detection with CRISPR-CaS13a/C2C2. Science. 2017;356:438-42.

32. Strich JR, Chertow DS. CRISPR-Cas biology and its application to infectious diseases. J Clin Microbiol. 2019;57:e01307-18.

33. Kuehn BM. Wearable biosensors studied for clinical monitoring and treatment. JAMA. 2016;316:255-7.

34. Seme K, Poljak M, Babič DZ, Močilnik T, Vince A. The role of core antigen detection in management of hepatitis C: a critical review. J Clin Virol. 2005;32:92-101.

35. van Tilborg M, Al Marzooqi SH, Wong WWL, Maan R, Vermehren J, Maasoumy B, et al. HCV core antigen as an alternative to HCV RNA testing in the era of directacting antivirals: retrospective screening and diagnostic cohort studies. Lancet Gastroenterol Hepatol. 2018;3:856-64.

36. Greenman J, Roberts T, Cohn J, Messac L. Dried blood spot in the genotyping, quantification and storage of HCV RNA: a systematic literature review. J Viral Hepat. 2015;22:353-61.

37. Soulier A, Poiteau L, Rosa I, Hezode C, Roudot-Thoraval F, Pawlotsky JM, et al. Dried blood spots: a tool to ensure broad access to hepatitis $C$ screening, diagnosis, and treatment monitoring. J Infect Dis. 2016;213:1087-95.

38. Lange B, Cohn J, Roberts T, Camp J, Chauffour J, Gummadi N, et al. Diagnostic accuracy of serological diagnosis of hepatitis $C$ and $B$ using dried blood spot samples (DBS): two systematic reviews and meta-analyses. BMC Infect Dis. 2017;17:700.

39. Lange B, Roberts T, Cohn J, Greenman J, Camp J, Ishizaki A, et al. Diagnostic accuracy of detection and quantification of HBV DNA and HCV RNA using dried blood spot (DBS) samples: a systematic review and meta-analysis. BMC Infect Dis. 2017;17:693.

40. Poljak M, Šterbenc A. Use of drones in clinical microbiology and infectious diseases: current status, challenges and barriers. Clin Microbiol Infect. 2020;26: 425-30.

41. Priye A, Wong S, Bi Y, Carpio M, Chang J, Coen M, et al. Lab-on-a-drone: toward pinpoint deployment of smartphone-enabled nucleic acid-based diagnostics for mobile health care. Anal Chem. 2016;88:4651-60.

42. Boutilier JJ, Brooks SC, Janmohamed A, Byers A, Buick JE, Zhan C, et al. Optimizing a drone network to deliver automated external defibrillators. Circulation. 2017;135:2454e65.

43. Lazarus JV, Wiktor S, Colombo M, Thursz M. EASL International Liver Foundation. Micro-elimination-a path to global elimination of hepatitis C. J Hepatol. 2017;67:665-6.

44. Haseltine WA. Universal disease screening and treatment-the Egyptian example. N Engl J Med. 2020;382:1081-3.

45. Waked I, Esmat G, Elsharkawy A, El-Serafy M, Abdel-Razek W, Ghalab R, et al. Screening and treatment program to eliminate hepatitis C in Egypt. N Engl J Med. 2020;382:1166-74.

46. Tsertsvadze T, Gamkrelidze A, Chkhartishvili N, Abutidze A, Sharvadze L, Kerash vili $V$, et al. Three years of progress towards achieving hepatitis $C$ elimination in the country of Georgia, April 2015 - March 2018. Clin Infect Dis. 2019;ciz956.

47. Averhoff F, Shadaker S, Gamkrelidze A, Kuchuloria T, Gvinjilia L, Getia V, et al. Progress and challenges of a pioneering hepatitis $C$ elimination program in the country of Georgia. J Hepatol. 2020;72:680-7.

48. Elgharably A, Gomaa Al, Crossey MME, Norsworthy PJ, Waked I, Taylor-Robinson SD. Hepatitis C in Egypt-past, present, and future. Int J Gen Med. 2016;10:1-6. 